\title{
PTS Sub-optimum Improved Algorithm Reducing PAPR of OFDM System
}

\author{
Sicong $\mathrm{He}^{1, \mathrm{a}}$, Guanghui Cai ${ }^{2, \mathrm{~b}}$ \\ ${ }^{1}$ School of Information Science and Engineering, Yunnan University, Kunming 650500, China; \\ ${ }^{2}$ School of Information Science and Engineering, Yunnan University, Kunming 650500, China; \\ a453845149@qq.com, bhcai@ynu.edu.cn
}

\section{Keywords: OFDM PAPR PTS APTS}

\begin{abstract}
For the problem of high PAPR in the Orthogonal Frequency Division Multiplexing (OFDM) system, Partial Transmission Sequence (PTS) algorithm is an effective method. However, the main problem of PTS algorithm is how to reduce computing complexity when searching the optimum phase. In this case, this paper brought about the Adaptive Partial Transmission Sequence (APTS) algorithm to reduce computing complexity. Based on the iteration in the APTS algorithm, the algorithm has completed when the computed PAPR is smaller than the set threshold value. The simulation results indicate that the adaptive PTS algorithm has a better performance than PTS algorithm in terms of the computing complexity.In other words, the computing complexity is smaller.
\end{abstract}

\section{Introduction}

As the high speed transmission technology of wireless channel, the OFDM, which has the orthogonal sub-carrier spectrum, can be partly overlapped. During the demodulation, the receiving end can recover the data from the sub-carrier, which those overlapped carriers are not orthogonal transformation. Therefore, increasing the utilization of the frequency spectrum significantly. 4G (The 4th Generation Mobile Communication Technology) technology is the mainstream of communication technology, and one of its core technologies is OFDM (Orthogonal Frequency Division Multiplexing) technology. OFDM is applied to the areas including Digital Audio Broadcasting (DAB), Digital Video Broadcasting (DVB), Asymmetric Digital Subscriber Line (ADSL), and Wireless Local Area Network (WLAN) ${ }^{[1]}$.

However, there is an obvious shortcoming of OFDM technology. It means that when the dynamic range of signal amplitude is oversized, signal peak power become much larger than the average and leading to a high PAPR value. The PAPR suppression technology includes: limiting technology, coding technology, scrambling technology, adaptive pre-distortion technology, and DFT spread spectrum technology. The PTS algorithm of scrambling technology can effectively reduce the PAPR value of the signal ${ }^{[2]}$.

CDF, used to reduce PAPR, is the common index of Technologies. It has always been replaced by CCDF in literature ${ }^{[1][2][6]}$. CCDF is the probability beyond certain threshold value PAPR $_{0}$. According to the central limit theorems, for the OFDM system with large sub-carrier quantity, it generate the real part and imaginary part of the OFDM time domain values, which meet the Gaussian distribution that the average value is 0 and variance is 0.5 . The amplitude of the multi-carrier meets Rayleigh distribution, with its power meets average value of 0 , and freedom degree of square $\chi^{2}$ of 2 . Then the CDF of the signal is:

$$
F(\mathrm{z})=1-\exp (-\mathrm{z})
$$

When using Nyquist sampling rate, the CCDF of the system can be expressed as:

$$
P(\operatorname{PAPR} \geq \mathrm{z})=1-\mathrm{P}(\mathrm{PAPR} \leq \mathrm{z})=1-\mathrm{F}(\mathrm{z})^{N}=1-[1-\exp (-\mathrm{z})]^{N}
$$




\section{Partial Transmission Sequence Algorithm}

The basic idea of part of transmission sequence is to enter n OFDM signal data to block X, and then dividing the block $\mathrm{X}$ into $\mathrm{V}$ sub-blocks without intersection.

$$
\mathrm{X}=\left[\mathrm{X}^{1}, \mathrm{X}^{2}, \ldots, \mathrm{X}^{\mathrm{V}}\right]^{\mathrm{T}}(3)
$$

Among the sub-blocks, $X^{i}$ refers to the continually subscribed sub-block in the same size. To reduce the PAPR, multiply the divided sub-blocks and phase factors. The phase factor is expressed as:

$$
b^{v}=e^{j \varphi^{v}}, e^{j \varphi^{v}} \in[0,2 \pi)
$$

After weighted, perform the IFFT, then:

$$
x=\operatorname{IFFT}\left\{\sum_{v=1}^{v} b^{v} X^{v}\right\}=\sum_{v=1}^{v} b^{v} \cdot \operatorname{IFFT}\left\{X^{v}\right\}=\sum_{v=1}^{v} b^{v} x^{v}
$$

Select several different phase factors $b^{v}$ to weight, calculate PAPR, and select the smallest PAPR $b^{v}$ of the phase factors

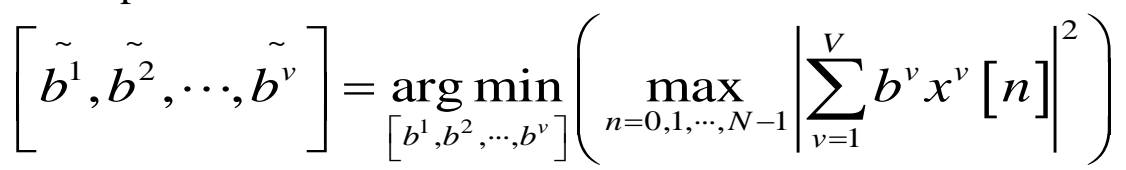

Then the time domain signal of the smallest PAPR vector can be expressed as:

$$
\tilde{x}=\sum_{v=1}^{V} \tilde{b}^{v} x^{v}
$$

The schematic block diagram of part transmission sequence is shown in figure $1^{[3]}$ :

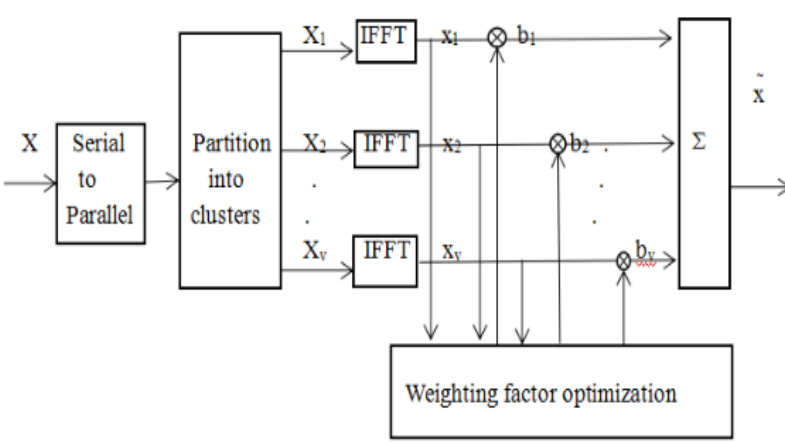

Figure 1 PTS system conceptual diagram

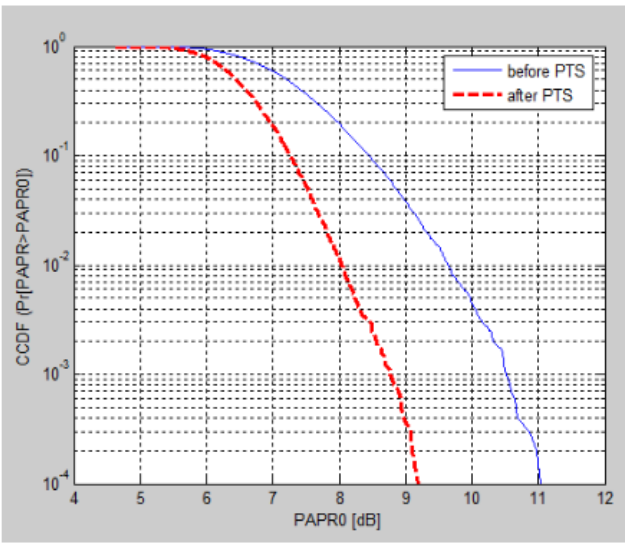

Figure 2 PAPR before and after PTS

When sub-block $\mathrm{V}=4$, sub-carrier $\mathrm{K}=128$, and phase factor is $b^{v} \in\{ \pm 1\}$, adopting the QPSK modulation. Figure 2 shows the CCDF of comparison of the PAPR performance curves between the PTS-OFDM system and the original OFDM system.

As is shown in figure 2, after PTS, the PAPR performance of the PTS-OFDM system is lower than the original OFDM system. PAPR has been suppressed effectively.

\section{Adaptive PTS Improvement Algorithm}

To select a technology to suppress PAPR, various factors should be considered, such as the ability to reduce PAPR, computing complexity and increasing of signal power. Specifically, the 
computing complexity is an important evaluation criterion to select an algorithm that can reduce PAPR ${ }^{[2]}$.For the algorithm that is good at reducing PAPR, its complexity is also higher. For example, in traditional PTS algorithm, all phase factors need to be explored in order to solve the optimum phase combination. When the numbers of group $\mathrm{V}$ increases, the computed quantity is $\mathrm{W}^{\mathrm{V}-1}$. Therefore, when selecting improved algorithm to reduce PAPR, the effect of reducing PAPR and computing complexity should be taken into consideration to obtain a relative better algorithm.

When the quantity of sub-blocks increases, there is a problem that the complexity of searching the optimum phase of traditional PTS algorithm is higher. The computed quantity will reach $\mathrm{W}^{\mathrm{V}-1}$, which is difficult to implement. This paper puts forward an adaptive PTS sub-optimum algorithm to reduce computing complexity, obtain a sub-optimum solution, and implement the simulation of the algorithm.

The core concept of adaptive PTS improved algorithm is to set a threshold value L of the PAPR and reduce the searching quantity by the threshold L. When the computed PAPR is smaller than the threshold $\mathrm{L}$, the iteration has completed and will not continue to the upper bound of $2^{\mathrm{V}-1}$. However, if the value of $\mathrm{L}$ is too small, the algorithm will search all factor combinations, then the final operation times will be close to PTS algorithm and not reduced. Apparently, the algorithm is unable to improve the operation speed; if the value of $\mathrm{L}$ is too large, the algorithm will search a small part of the factor combinations to obtain a sub-optimum solution that differs from the optimum solution. Therefore, the threshold value L should be the compromise of PAPR suppression and computing complexity.

The algorithm is as followed:

1.Select the threshold $L$ of PAPR, the iteration quantity $K$ in the range of $1 \leqslant K \leqslant 2^{\mathrm{V}-1}$;

2.Divide the entered $\mathrm{N}$ sub-carriers into $\mathrm{V}$ sub-blocks, the initial value of phase factor: $b^{\mathrm{v}}=1, \mathrm{v}=1,2,3 \ldots \mathrm{V}$;

3.Set a as the computing quantity, with initial value of 1 . Compute the PAPR;

4.If PAPR $<\mathrm{L}$ or $\mathrm{a}>\mathrm{K}$, the iteration ends; otherwise if PAPR $>\mathrm{L}$ or $\mathrm{a}<\mathrm{K}$, change the original $b^{\mathrm{v}}=1$, and set $b^{\mathrm{v}}=-1$, and $\mathrm{a}=\mathrm{a}+1$. The circulation ends until the $\mathrm{M}$ factor in the phase factor changes. Select the phase factor corresponds to the smallest PAPR.

The flowchart of the adaptive PTS sub-optimum improved algorithm is shown in figure 3:

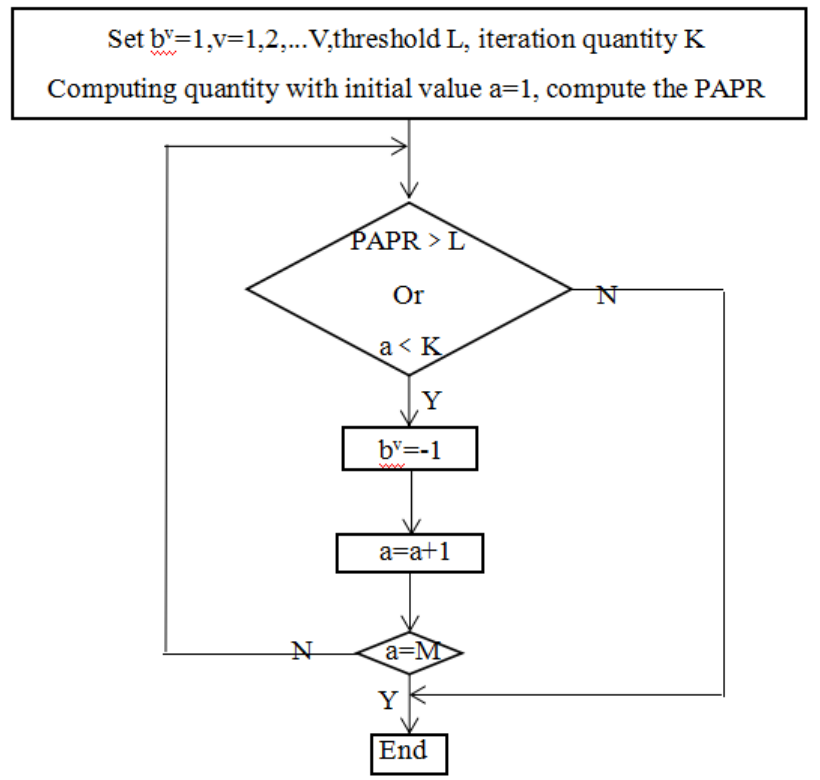

Figure 3 APTS algorithm flowchart

The concept of threshold comes from the adaptive PTS sub-optimum improved algorithm. From the result of simulation, if the threshold is appropriate, the computing complexity will be reduced, 
i.e. $\mathrm{V} \leqslant \mathrm{W}^{\mathrm{V}-1}$, and a sub-optimum solution will be obtained.

Figure 4 shows different PAPR suppression effects under the same conditions that sub-block quantity $\mathrm{V}=4$, sub-carrier $\mathrm{K}=128$. When adopting QPSK modulation, OFDM, original PTS, adaptive PTS sub-optimum improvement $\mathrm{L}=7$ (A-PTS1), and adaptive PTS sub-optimum improvement L=6.5(A-PTS2) present different effects.

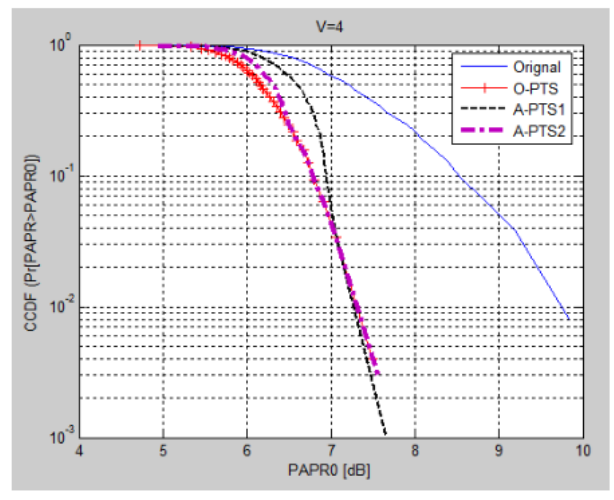

Figure 4 PAPR of Original PTS and APTS

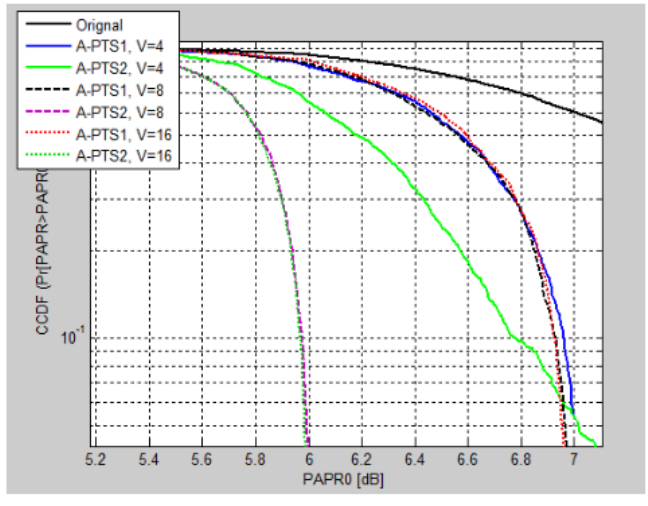

Figure 5 PAPR of Original PTS and APTS with various V

As is shown in figure 4, PTS algorithm and APTS algorithm have the effect on PAPR suppression.

Based on the simulation, the computing quantity of APTS algorithm is significantly smaller than the traditional PTS algorithm. In the APTS algorithm, when the threshold value equals 7 , the search quantity through simulation: average_k=2.9770, average time $=3$; when $\mathrm{L}$ equals 6.5 , search quantity average_k_comp1 $=6.5290$, and average time $=7$, while the search time in the traditional PTS algorithm is $2^{4-1}=8$, which means the computing quantity of the APTS algorithm is smaller than the traditional PTS algorithm.

Different threshold values also have different effects on the computing complexity. When the threshold $\mathrm{L}=7$, the search quantity of the improved algorithm is $62.5 \%$ less than the original search quantity; when $\mathrm{L}=6.5$, the search quantity of the improved algorithm is $12.5 \%$ less than the original algorithm. In other words, the computing quantity is significantly reduced, with enhanced computing speed.

Figure 5 shows when sub-carrier $\mathrm{K}=128$, and adopting QPSK modulation,the CCDF of OFDM,and the PAPR suppression of adaptive PTS sub-optimum improvement L=7(A-PTS1), and adaptive PTS sub-optimum improvement L=6(A-PTS2) with sub-block quantities V=4,8 and 16 .

As is shown in figure 5,when the quantity of sub-block increases, the PAPR suppression effect of APTS algorithm is continually improved.Meanwhile,the computing quantity of APTS algorithm is constantly reduced,and the computing speed is continuously enhanced.

Table 1 shows the comparison of PTS iteration quantity and APTS (when L $=7$ ) iteration quantity when sub-carrier $\mathrm{K}=128$, and sub-block quantity $\mathrm{V}$ values 4,8 , and 16 in QPSK modulation.

Table 1 Iteration quantity of APTS algorithm and PTS algorithm

\begin{tabular}{|c|c|c|c|}
\hline Sub-block & $\begin{array}{c}\text { PTS iteration } \\
\text { quantity }\end{array}$ & $\begin{array}{c}\text { APTS iteration } \\
\text { quantity }\end{array}$ & $\begin{array}{c}\text { Comparison of } \\
\text { APTS and PTS } \\
\text { iteration quantity }\end{array}$ \\
\hline 4 & 8 & 3 & $62.5 \%$ \\
\hline 8 & 128 & 28 & $78.1 \%$ \\
\hline 16 & 32768 & 36 & $99.8 \%$ \\
\hline
\end{tabular}

When the quantity of sub-block increases, the search quantity of the PTS algorithm is $2^{\mathrm{v}-1}$, 
increased in exponent. Take the condition that $\mathrm{V}=4, \mathrm{~V}=8$, and $\mathrm{V}=16$ for example. When $\mathrm{V}=4$, the search times of PTS algorithm is 8 while the search times of APTS algorithm is 3. Compared to the PTS algorithm, the search times of APTS reduced by $62.5 \%$. When $\mathrm{V}=8$, the search times of PTS algorithm is128, however, it is only 28 times of APTS algorithm's search times. Significantly, there is a $78.1 \%$ decrease; when V $=16$, the search times of APTS algorithm is 36, at the meanwhile, the search times of PTS algorithm reach 32768, The APTS algorithm is $99.8 \%$ lower than the PTS algorithm. It is obvious that the computing complexity decreased a lot. When the quantity of sub-block continually increases, the advantage of improved algorithm, which reduce the operation times, will be more and more significant.

\section{Summary}

Currently, PTS, which reduces the high PAPR of OFDM system, has the disadvantage of high computing complexity. It needs to search all phase factors to find the optimum solution in $\mathrm{W}^{\mathrm{V}-1}$ times of computing. This paper put forward the APTS sub-optimum improved algorithm that well solved the computing complexity issue, which eliminates the unnecessary loop in the PTS algorithm. When V increases, the computing quantity of PTS also increases in exponent, however, APTS only needs $\mathrm{V}$ times computing, and we can see that $\mathrm{V} \leqslant \mathrm{W}^{\mathrm{V}-1}$ obviously.Based on the simulation,the obtained sub-optimum solution of APTS sub-optimum improved algorithm is close to the optimum solution. In this way, the high PAPR and computing complexity are both suppressed.

\section{Acknowledgements}

This work was financially supported by the Yunnan Education Department Science Foundation (2014Y020)

\section{Reference}

[1]Jiang Tao.The research of PAPR of OFDM wireless communication system [D]. Doctor. Wuhan. Huazhong University of Science and Technology.2004

[2] Wang Fang.The algorithm research of PAPR suppression of OFDM system [D]. Master.Hebei. Hebei University.2010,p.20-21

[3] Xing Gang,Li Hui and Gao Weiting.The PTS iteration search algorithm of reducing PAPR of OFDM system[J].Computer Engineering and Applications.2013(10)

[4] Wu Jiao,Yang Hongwen and Zhang Xin.A new block iteration PTS algorithm of OFDM system[J].Journal of electronics and information.2008(12)

[5] Cao Ruoyun and Lin Wei.An adaptive redundant sequence algorithm of reducing PAPR of OFDM signals[J].Modern electronic technology.2008(17)

[6] Han yan.The algorithm research of reducing PAPR of OFDM system[D].Master.Xi'an.Xi'an Electronic Science and Technology University.2010

[7] A.D.S.Jayalath and C.Tellambura. Adaptive PTS approach for reduction of peak-to-average power ratio of OFDM signals[J].ELECTRONICS LETTERS.2000(14),p.1226-1228

[8] Jun Hou,Jianhua Ge,Jing Li. Peak-To-Average Power Ratio Reduction of OFDM Signals Using PTS Scheme With Low Computational Complexity[J].IEEE TRANSACTIONS ON BROADCASTING.2011(1),p.143-147 